CPTH-A193.0892

\title{
FRACTIONALLY CHARGED PARTICLES \\ AND SUPERSYMMETRY BREAKING IN 4D STRINGS
}

\author{
I. Antoniadis and K. Benakli \\ Centre de Physique Théorique \\ Ecole Polytechnique, 91128 Palaiseau, France
}

\begin{abstract}
Four-dimensional string theories predict in general the existence of light exotic particles with fractional electric charges. Such particles could escape present observations if they are confined by a gauge group of the "hidden" sector into integrally charged states. It is conceivable that the same gauge group is also responsible for dynamical supersymmetry breaking, via gaugino and scalar condensation. The communication of the breaking to the observable sector is now mediated by ordinary gauge interactions, implying that the confining scale can be in the TeV-region.

We study the main phenomenological implications of this possibility. In particular, we analyze the pattern of supersymmetry breaking and the mass-spectrum of the sparticles. We also show that this scenario can be consistent with the unification of all coupling constants at the string scale.
\end{abstract}

CPTH-A193.0892

August 1992 
A main issue in string theory is to derive a four-dimensional model, which leads, at low energies, to the Standard Model of electroweak and strong interactions. Although there is a lot of arbitrariness due to the infinite vacuum degeneracy, there are still many stringy constraints emerging from the internal consistency of the theory. Here, we discuss two main implications related to the generic existence of particles with fractional electric charges [1-3] and to the problem of dynamical supersymmetry breaking.

One important restriction is on the allowed matter representations. Given a simple gauge group factor $G_{i}$, the dimensions of the possible unitary massless representations are constrained by the level $k_{i}$ of the associated Kac-Moody algebra on the world-sheet. The positive integer paremeter $k_{i}$ determines also the corresponding tree-level gauge coupling constant $\alpha_{i}$, in terms of the four-dimensional string coupling $\alpha, \alpha_{i}=\alpha / k_{i}$. Models with $k_{i}>1$ are difficult to construct and they lead in general to "exotic" representations in the massless spectrum, e.g. $S U(3)$-octets or $S U(2)$-triplets or higher. In contrast, models with $k_{i}=1$ are simpler, and they guarantee that the only possible massless representations are singlets or doublets of $S U(2)$ and singlets or triplets of $S U(3)$. More generally, they allow vectors and spinors of orthogonal groups or antisymmetric representations of unitary groups. Moreover, they imply an automatic unification of all couplings at the Planck scale $M_{p}$. However, in this case, it is impossible to impose the value for the weak angle $\sin ^{2} \theta_{w}=\frac{3}{8}$ at $M_{p}$ similtaneously with the observed electric charge quantization at the level of the string-derived Standard Model [3]. Thus, unless the Weinberg angle at $M_{p}$ is too small, we must deal with fractional electric charged particles (FEC-particles). In general, a weaker charge quantization condition can be imposed depending on the compactification; for instance, that color neutral states have charges multiples of $1 / 2$ for fermionic constructions, or $1 / N$ for $Z_{N}$ orbifolds [4].

The lightest FEC-particle must be stable. Previous searches of such particles lead to several bounds on their masses provided their charges are not ridiculously small [5]. On the one hand, masses below approximately $100 \mathrm{GeV}$ are excluded by accelerator experiments. On the other hand, masses above a few $\mathrm{TeV}$ would overclose the Universe and are also excluded, leaving a "window" for masses of a few hundred $\mathrm{GeV}$. However, for these masses, an estimation of their relic abundancies seems to contradict the upper bounds found in Millikan experiments by several orders of magnitude [2]. Therefore, free light FEC-particles are excluded, unless there is some unkown mechanism which supresses their abundance on earth. There 
remain two possible ways to escape the experimental constraints. The first is to make them superheavy, with masses of the order $M_{p}$. The second and most natural is to confine them by a gauge group of the hidden sector into integrally charged bound states like the quarks in QCD [6].

Another important problem is that of supersymmetry breaking, which must occur at energies of the order of the weak-interaction scale, to protect the gauge hierarchy. A popular scenario for its attractive features is based on gaugino condensation in a gauge group of the hidden sector, at a high scale $\Lambda \sim 10^{13} \mathrm{GeV}$ [7]. The supersymmetry breaking is communicated to the observable sector by gravitational interactions, giving rise to small mass-splittings for the superpartners of the order of $\frac{\Lambda^{3}}{M_{p}^{2}} \sim 1 \mathrm{Tev}$.

In this letter, we explore the idea that the gauge group $G$ which confines the fractionally charged particles is also responsible for the breaking of supersymmetry. In any case, the existence of a group which confines the FEC-particles could always provide an additional source of supersymmetry breaking via non-perturbative condensate formation of gauginos or scalar components of chiral supermultiplets. The latter could give rise to mass-splittings in the "hidden" sector, in particular between the components of the fractionally charged matter supermultiplets, of the order of the confinement scale $\Lambda$ through dimension-four renormalizable interactions. Since the breaking is now communicated to the "observable" sector by strong and electroweak interactions, $\Lambda$ must be of the order of a few TeV. This may be a generic feature of a large class of models. In this work, we study the main phenomenological implications of this mechanism. In particular, we analyze the novel pattern of supersymmetry breaking, the mass-spectrum of the sparticles and the modifications to the coupling constant unification. This scenario also predicts a rich spectrum of new composite particles in the TeV-region, called "cryptons" [8].

\section{SPARTICLE MASSES}

To simplify the discussion, we restrict our analysis to the following content of the "hidden" sector:

-The gauge group $G$ is $S O(N)$ or $S U(N)$.

-The FEC-particles are in vector representations of $G: N$ and $\bar{N}$ of $S U(N)$ or $N$ of $S O(N)$ with a common mass-splitting $\delta M$ between scalars and fermions superpartners. Moreover, these particles transform non trivially under the Standard 
Model gauge group. The allowed representations are denoted by $Q \equiv\left(3,2, \frac{e_{Q}}{3 N}\right)$, $D \equiv\left(\overline{3}, 1, \frac{e_{D}}{3 N}\right), L \equiv\left(1,2, \frac{e_{L}}{N}\right), E \equiv\left(1,1, \frac{e_{E}}{N}\right)$, under $S U(3) \times S U(2)$ with the third position inside the brackets denoting the electric charge (in the case of doublet it is the charge of the upper component). The numbers $e_{Q, D, L, E}$ must be integers, so that gauge singlet bound states of $G \times S U(3)$ are integrally charged. We also allow for different multiplicities $n_{Q}, n_{D}, n_{L}$ and $n_{E}$ associated to the pairs of chiral-antichiral representations $Q+\bar{Q}, D+\bar{D}, L+\bar{L}$ and $E+\bar{E}$, respectively.

Supersymmetry breaking is communicated to the observable sector through gauge interactions. In fact, radiative corrections in the sparticle propagators involving the exhange of FEC-fields induce mass-splittings in the usual Standard Model supermultiplets. Below, we evaluate these contributrions to the lowest order.

We first consider the gaugino masses $m_{\tilde{g}_{i}}$, which receive contributions from the one-loop graphs involving the exchange of the fermion and scalar components of all FEC-supermultiplets. They were computed in Ref.[9] with the result:

$$
m_{\tilde{g}_{i}}=\sum_{j} c_{\tilde{g}_{i}} M_{j} F\left(\frac{\delta M}{M_{j}}\right)
$$

where $c_{\tilde{g}_{i}}$ is a constant factor depending on the gauge group and matter representations, while $F$ is a function which depends only on the ratio of the mass-splitting $\delta M$ over the supersymmetric mass $M_{j}$ of the $j$-th FEC-multiplet:

$$
F(x)=-\frac{(1+x)^{2}}{1-(1+x)^{2}} \ln \left((1+x)^{2}\right)+\frac{(1-x)^{2}}{1-(1-x)^{2}} \ln \left((1-x)^{2}\right) .
$$

The function $\frac{1}{x} F(x)$ is nearly constant for $x \lesssim 1$ and it goes to zero asymptotically as $1 / x$ for $x \gtrsim 2$. In order to avoid too small gaugino masses, we concentrate on the region $M_{j} \gtrsim \delta M$, where we can use the following approximation :

$$
M_{j} F\left(\frac{\delta M}{M_{j}}\right) \simeq 2 \delta M
$$

The gaugino masses are then independent of the supersymmetric masses of the

FEC-particles and they are proportional to the mass-splitting $\delta M$.

The explicit formulas for gluinos, wino and the two neutralinogauginos soft masses are: 


$$
\begin{aligned}
& m_{\tilde{g}_{3}}=\frac{\alpha_{s}}{8 \pi} 4 N \delta M\left(2 n_{Q}+n_{D}\right) \\
& m_{\tilde{g}_{2}}=\frac{\alpha_{e m}}{4 \pi} 2 N \frac{\delta M}{\sin ^{2} \theta_{W}}\left(3 n_{Q}+n_{L}\right) \\
& m_{\tilde{g}_{1}}=\frac{\alpha_{e m}}{4 \pi} \frac{4}{N} \frac{\delta M}{\cos ^{2} \theta_{W}}\left(n_{Q} \frac{\left(2 e_{Q}-3 N\right)^{2}}{6}+n_{D} \frac{e_{D}^{2}}{3}+n_{L} \frac{\left(2 e_{L}-N\right)^{2}}{2}+n_{E} e_{E}^{2}\right) .
\end{aligned}
$$

We now consider squark and slepton masses. These are in general model dependent because they also depend on possible superpotential terms among ordinary matter and FEC-particles. To get a rough estimate for the various orders of magnitude, we calculate the contributions to scalar masses from radiative corrections of gauge interactions. The contribution of tadpoles, which involve the exchange of FEC-scalars coming from the $U(1)$-hypercharge D-term, vanishes in our case, since we consider left-right symmetric representations with a common mass-splitting $\delta M$. The leading contribution are two-loop diagrams involving one loop of Standard Model particles and one loop of FEC particles [10]. These diagrams are logarithmically divergent and require the use of some ultraviolet cut-off, corresponding to the higher scale up to which the effective field theory remains valid. Replacing the cutoff with the string unification scale $M_{\mathrm{SU}}$, the computation of radiative corrections to the squark and slepton masses give [11]:

$$
\begin{aligned}
\delta m_{\tilde{u}_{L}}^{2} & =\frac{4}{3} m_{3}^{2}+\frac{3}{4} m_{2}^{2}+\frac{1}{36} m_{1}^{2} \\
\delta m_{\tilde{d}_{L}}^{2} & =\frac{4}{3} m_{3}^{2}+\frac{3}{4} m_{2}^{2}+\frac{1}{36} m_{1}^{2} \\
\delta m_{\tilde{u}_{R}}^{2} & =\frac{4}{3} m_{3}^{2}+\frac{4}{9} m_{1}^{2} \\
\delta m_{\tilde{d}_{R}}^{2} & =\frac{4}{3} m_{3}^{2}+\frac{1}{9} m_{1}^{2} \\
\delta m_{\tilde{e}_{L}}^{2} & =\frac{3}{4} m_{2}^{2}+\frac{1}{4} m_{1}^{2} \\
\delta m_{\tilde{\nu}_{L}}^{2} & =\frac{3}{4} m_{2}^{2}+\frac{1}{4} m_{1}^{2} \\
\delta m_{\tilde{e}_{R}}^{2} & =m_{1}^{2},
\end{aligned}
$$


with

$$
\begin{aligned}
m_{1}^{2} & =\left(\frac{\alpha_{e m}}{\pi}\right)^{2} \frac{1}{N^{3}} \frac{\delta M^{2}}{\cos ^{4} \theta_{W}}\left(n_{Q} \frac{\left(2 e_{Q}-3 N\right)^{4}}{216} \ln \left(\frac{M_{\mathrm{SU}}}{M_{Q}}\right)+n_{D} \frac{e_{D}^{4}}{27} \ln \left(\frac{M_{\mathrm{SU}}}{M_{D}}\right)\right. \\
& \left.+n_{L} \frac{\left(2 e_{L}-N\right)^{4}}{8} \ln \left(\frac{M_{\mathrm{SU}}}{M_{L}}\right)+n_{E} e_{E}^{4} \ln \left(\frac{M_{\mathrm{SU}}}{M_{E}}\right)\right) \\
m_{2}^{2} & =\left(\frac{\alpha_{e m}}{\pi}\right)^{2} N \frac{\delta M^{2}}{\sin ^{4} \theta_{W}}\left(3 n_{Q} \ln \left(\frac{M_{\mathrm{SU}}}{M_{Q}}\right)+n_{L} \ln \left(\frac{M_{\mathrm{SU}}}{M_{L}}\right)\right) \\
m_{3}^{2} & =\left(\frac{\alpha_{s}}{\pi}\right)^{2} N \delta M^{2}\left(2 n_{Q} \ln \left(\frac{M_{\mathrm{SU}}}{M_{Q}}\right)+n_{D} \ln \left(\frac{M_{\mathrm{SU}}}{M_{D}}\right)\right)
\end{aligned}
$$

where $\alpha_{i}, i=1,2,3$ are the Standard Model coupling constants of $U(1), S U(2)$ and $S U(3)$, respectively. In (5) we have not included the contribution coming from the Higgs sector, which is model dependent and will be discussed below. Moreover, in the absence of Yukawa couplings, the scalar masses are the same for all families. Numerical examples for the above sparticle masses will be given at the end.

\section{COUPLING CONSTANT UNIFICATION}

The existence of light fractionally charged particles could alter significantly the coupling constant unification. In fact, at the one loop level, the gauge couplings at the energy scale $Q$ depend on the particle content through the renormalization group formula:

$$
\frac{1}{\alpha_{i}(Q)}=\frac{1}{\alpha_{\mathrm{GUT}}}+\frac{b_{i}}{2 \pi} \ln \left(\frac{M_{\mathrm{GUT}}}{Q}\right)
$$

where $M_{\mathrm{GUT}}$ is the unification scale, and $b_{i}$ is the one-loop beta-function

$$
b_{i}=-3 C\left(G_{i}\right)+\sum_{\operatorname{reps} R_{i}} T\left(R_{i}\right)
$$

In (8) $C\left(G_{i}\right)$ is the quadratic Casimir of the group $G_{i}$ and equals $N$ for $S U(N)$ and $N-2$ for $S O(N) . T\left(R_{i}\right)$ is the index of the corresponding matter representation $R_{i}$; it is equal to $\frac{1}{2}$ for chiral supermultiplets in the fundamental representation of $S U(N)$, while it is reduced to the sum of the squares of charges in the case of $U(1)$-hypercharge. In the case of $S U(3) \times S U(2) \times U(1)$, one can solve for $M_{\mathrm{GUT}}$ and $\sin ^{2} \theta_{W}\left(m_{Z}\right)$ as a function of the strong and electromagnetic coupling constants 
$\alpha_{s}\left(m_{Z}\right)$ and $\alpha_{e m}\left(m_{Z}\right)$ at $m_{Z}$ :

$$
\begin{aligned}
& \ln \left(\frac{M_{\mathrm{GUT}}}{M_{Z}}\right)=\frac{\pi}{6}\left(\frac{3}{5 \alpha_{e m}\left(m_{Z}\right)}-\frac{8}{5 \alpha_{s}\left(m_{Z}\right)}\right)-0.21- \frac{1}{20}\left[\frac{L_{Q}}{6}\left(\left(\frac{2 e_{Q}-3 N}{N}\right)^{2}-7\right)\right. \\
&+\frac{L_{D}}{3}\left(\frac{e_{D}^{2}}{N^{2}}-4\right) \\
&+\frac{L_{L}}{2}\left(\frac{\left(2 e_{L}-N\right)^{2}}{N^{2}}+1\right) \\
&+\left.L_{E}\left(\frac{e_{E}^{2}}{N^{2}}\right)\right] \\
& \sin ^{2} \theta_{W}\left(m_{Z}\right)=0.0029+\frac{1}{5}+\frac{7 \alpha_{e m}\left(m_{Z}\right)}{15 \alpha_{s}\left(m_{Z}\right)}+\frac{\alpha_{e m}\left(m_{Z}\right)}{20 \pi}\left[\frac{L_{Q}}{3}\left(-\left(\frac{2 e_{Q}-3 N}{N}\right)^{2}+22\right)\right. \\
&-L_{D}\left(2\left(\frac{e_{D}^{2}}{3 N^{2}}-\frac{4}{3}\right)+5\right) \\
&+L_{L}\left(-\frac{\left(2 e_{L}-N\right)^{2}}{N^{2}}+4\right) \\
&\left.+L_{E}\left(-2 \frac{e_{E}^{2}}{N^{2}}\right)\right]
\end{aligned}
$$

where $L_{R} \equiv \sum_{i} \ln \left(\frac{M_{\mathrm{GUT}}}{m_{R_{i}}}\right)$ with the sum running over all supermultiplets $i$ in the representation $R . \alpha_{s}\left(m_{Z}\right), \alpha_{e m}\left(m_{Z}\right)$ and $\sin ^{2} \theta_{W}\left(m_{Z}\right)$ are defined in the $\overline{\mathrm{MS}}$ scheme, while the small numbers -0.21 and 0.0029 in the r.h.s. of (9) and (10) take into account two-loop corrections [12]. Substituting all $L_{R}=0$, the above equations take into account the particle spectrum of the minimal supersymmetric Standard Model. In this case, one obtains good agreement with the experimental value of the weak angle and a predicted value for $M_{\mathrm{GUT}} \sim 2 \times 10^{16} \mathrm{GeV}$ [13]. This value turns out to be more than one order of magnitude smaller than the string unification scale $M_{\mathrm{SU}} \sim 3.73 \times 10^{17} \mathrm{GeV}[14]$.

The existence of additional representations, besides those of the minimal supersymmetric Standard model, could move $M_{\mathrm{GUT}}$ close to $M_{\mathrm{SU}}$ without destroying the succesful prediction for $\sin ^{2} \theta_{W}\left(m_{Z}\right)$ if they satisfy the following inequalities:

$53 \lesssim\left[\frac{L_{Q}}{6}\left(7-\left(\frac{2 e_{Q}-3 N}{N}\right)^{2}\right)+\frac{L_{D}}{3}\left(4-\frac{e_{D}^{2}}{N^{2}}\right)-\frac{L_{L}}{2}\left(\frac{\left(2 e_{L}-N\right)^{2}}{N^{2}}+1\right)-L_{E}\left(\frac{e_{E}^{2}}{N^{2}}\right)\right] \lesssim 70$ 


$$
\begin{aligned}
-22 \lesssim\left[\frac{L_{Q}}{3}\left(-\left(\frac{2 e_{Q}-3 N}{N}\right)^{2}+22\right)\right. & -L_{D}\left(2\left(\frac{e_{D}^{2}}{3 N^{2}}-\frac{4}{3}\right)+5\right) \\
& \left.+L_{L}\left(-\frac{\left(2 e_{L}-N\right)^{2}}{N^{2}}+4\right)+L_{E}\left(-2 \frac{e_{E}^{2}}{N^{2}}\right)\right] \lesssim 7.5
\end{aligned}
$$

The above relations are obtained from (9) and (10), using the values $M_{\mathrm{GUT}}=$ $M_{\mathrm{SU}} \sim 3.73 \times 10^{17} \mathrm{GeV}, \alpha_{e m}\left(m_{Z}\right)=\frac{1}{128}, \sin ^{2} \theta_{W}\left(m_{Z}\right)=0.233$ and

$\alpha_{s}\left(m_{Z}\right)=0.118 \pm 0.007$ [15]. The inequalities (11) and (12) were studied in

Ref.[12] for the case of non-exotic and integrally charged Standard Model representations. These correspond to the values $\frac{e_{Q}}{N}=2, \frac{e_{D}}{N}=-2$ or $1, \frac{e_{L}}{N}=1$ and $\frac{e_{E}}{N}=-1$, which are associated to additional quark doublets, up or down antiquarks, leptons and antileptons, respectively. In this case, it was found that every solution of (11) and (12) requires the existence of at least one additional pair of quark doublets $Q+\bar{Q}$ with mass much smaller than $M_{\mathrm{SU}}$. This follows from the combination $3 \times$ $(11)+(12)$, which reads:

$$
27 \lesssim\left[\frac{L_{Q}}{6}\left(13-\left(\frac{2 e_{Q}}{N}-3\right)^{2}\right)+\frac{L_{D}}{3}\left(1-\frac{e_{D}^{2}}{N^{2}}\right)+\frac{L_{L}}{2}\left(1-\left(\frac{2 e_{L}}{N}-1\right)^{2}\right)-\frac{e_{E}^{2}}{N^{2}} L_{E}\right] \lesssim 44 .
$$

For the above mentioned values, the coefficients of all terms in (13) become negative except the one of $L_{Q}$, implying $L_{Q} \neq 0$. However, in the presence of FEC-particles the coefficient of $L_{L}$ is also positive and more general solutions exist [16].

At the string unification scale $M_{\mathrm{SU}}$ the coupling constant of the gauge group $G$ is also unified with the three Standard Model couplings and is equal to $\alpha_{\mathrm{GUT}}$. This leads to an additional constraint on the matter content of $G$, emerging from the requirement that the confinement scale $\Lambda$ must have the desired value. A necessary condition is that when taking into account just the FEC-states in the renormalization group equation for the $G$ coupling constant in (7) and (8), the resulting confinement scale $\Lambda_{\text {FEC }}$ verifies:

$$
\Lambda_{\mathrm{FEC}} \gtrsim \delta M \sim \mathrm{TeV}
$$

\section{NUMERICAL EXAMPLES}

Here, we work out some explicit numerical examples to show that the various masses of fractionally charged supermultiplets, chosen appropriately, can lead to a 
reasonable spectrum of ordinary sparticles in the region of a hundred $\mathrm{GeV}$. At the same time we pay attention to be consistent with the perturbative unification of all couplings at the string scale, which implies in particular that the inequalities (11), (12) and (14) must hold.

The choice of the "hidden" group $G$ has to satisfy the observed charge quantization that singlet bound states of $S U(3) \times G$ must have integer electric charges. For $G=\prod_{N} S U(N) \times \prod_{n} S O(2 n)$,

this reduces to the following condition (for Kac-Moody levels $k=1$ ) [3],[8]:

$$
\sum_{N} \frac{i_{N}\left(N-i_{N}\right)}{2 N}+\sum_{n}\left\{\begin{array}{ll}
0 & \text { for } j_{n}=0 \\
1 / 2 & \text { for } j_{n}=2 \\
n / 8 & \text { for } j_{n}=1
\end{array}=\right.\text { non zero integer, }
$$

where for every $N, i_{N}$ is some integer between 0 and $N-1$. Moreover, the electric charge of a state transforming in the representation $N$ or $\bar{N}$ of $S U(N)$ and/or $2 n$ of $S O(2 n)$ is given by:

$$
q= \pm \sum_{N} \frac{i_{N}}{N}+\sum_{n} \frac{j_{n}}{2} \bmod 1
$$

where the \pm sign stands for the representations $N$ or $\bar{N}$, and the integers $i_{N}$ and $j_{n}$ are those which satisfy (15).

In the case where $G$ is semi-simple, the smallest group satisfying (15) is $S O(16)$ (with $j_{8}=1$ ) for orthogonal groups or $S U(8)$ (with $i_{8}=4$ ) for the unitary ones. In both cases, the electric charges of FEC color-singlet states are half-integers. For such big groups one must be careful in the choice of the matter content, so that the value of $\alpha_{\mathrm{GUT}}$ is in the perturbative domain. Notice that the presence of even one representation of type $D+\bar{D}$, which is the minimum requirement for non-zero one-loop gluino masses, leads to an $S U(3)$ beta function $b_{3}=N-3$ becoming large and positive for large N. Perturbative unification then implies that the masses $M_{D}$ of these representations are very high. For example, $M_{D} \gtrsim 10^{14} \mathrm{GeV}$ for $S O(16)$, or $M_{D} \gtrsim 10^{10} \mathrm{GeV}$ for $S U(8)$.

When $G$ is a product of semi-simple factors many more solutions exist. Here, we examine an example, where $G=S U(4) \times S O(2 n)$ (with $i_{4}=2$ and $j_{n}=2$ ) which is a solution of (15) for every $n$ with half-integer electric charges for the FEC colorsinglet states. For simplicity we will assume that all FEC light states transform non-trivially only under $S U(4)$, which is for instance the case in the flipped $S U(5)$ model of Ref.[6] with hidden sector $S U(4) \times S O(10)$. In this case, perturbative unification implies no bounds on the masses $M_{D}$. 
Now, we derive the mass spectrum for an example with $S U(4)$ group. Using the values:

$$
\begin{aligned}
& M_{S U} \sim 3.7 \times 10^{17} \mathrm{GeV} \quad \delta M=15 \mathrm{TeV} \quad \text { confinement group : } S U(4) \times G, \\
& n_{Q}=1, e_{Q}=1, m_{Q}=3 \times 10^{14} \mathrm{GeV}, \quad n_{D}=2, e_{D}=1, m_{D}=2 \times 10^{15} \mathrm{GeV}, \\
& n_{L}=0, \quad n_{E}=1, e_{E}=3, m_{E}=10^{13} \mathrm{GeV} .
\end{aligned}
$$

we find:

\begin{tabular}{|c|c|}
\hline Sparticles & Masses (in GeV) \\
\hline gluinos & 298 \\
\hline charginos & 960 \\
\hline neutralinos & 320 \\
\hline$\delta m_{\tilde{u}_{L}}$ & 1350 \\
\hline$\delta m_{\tilde{d}_{L}}$ & 1350 \\
\hline$\delta m_{\tilde{u}_{R}}$ & 450 \\
\hline$\delta m_{\tilde{d}_{R}}$ & 450 \\
\hline$\delta m_{\tilde{e}_{L}}$ & 1290 \\
\hline$\delta m_{\tilde{\nu}_{L}}$ & 1290 \\
\hline$\delta m_{\tilde{e}_{R}}$ & 209 \\
\hline
\end{tabular}

As already mentioned above, the values of scalar masses do not include the Higgs, as well as possible superpotential contributions, which are model dependent. In the case of minimal supersymmetric standard model, the Higgs contribution, neglecting Yukawa couplings, represents small correction for heavy particles but potentially important correction for the light ones. Yukawa couplings are expected to modify mainly the stop masses.

A particular characteristic of this scenario is the large mass difference between squarks and sleptons, which is a consequence of the radiative origin of supersymmetry breaking communication to the observable sector. This difference is due to the ratio of the strong over the electroweak coupling constant and could be compensated only by the addition of many FEC-particles in representations of type $L$ and $E$. Another characteristic of this mechanism is that the right-selectron seems to be the lightest supersymmetric particle, although a more careful study of the Higgs sector is required for a definite conclusion. Finally, this class of models predicts new composite heavy particles with integer electric charges. The explicit construction of $4 \mathrm{D}$ string models with the desired properties described in this work remain of course an open problem. 


\section{REFERENCES}

[1] X. Wen and E. Witten, Nucl. Phys. B261 (1985) 651.

[2] G. Athanasiu, J. Atick, M. Dine and W. Fischler, Phys. Lett. 214B (1988) 55.

[3] A. Schellekens, Phys. Lett. 237B (1990) 363.

[4] I. Antoniadis, Proceedings of Summer School in High Energy Physics and Cosmology, Trieste (1990) 677.

[5] D. Bailey, B. Campbell and S. Davidson, Phys. Rev. D43 (1991) 2314.

[6] I. Antoniadis, J. Ellis, J. Hagelin and D.V. Nanopoulos, Phys. Lett. 231B (1989) 65.

[7] J.P. Derendinger, L.E. Ibáñez and H.P. Nilles, Phys. Lett. 155B (1985) 65; M. Dine, R. Rohm, N. Seiberg and E. Witten, Phys. Lett. 156B (1985) 55.

[8] J. Ellis, J.L. Lopez and D.V. Nanopoulos, Phys. Lett. 245B (1990) 375 and 247B (1990) 257.

[9] R. Barbieri, L. Girardello and A. Masiero, Phys. Lett. 127B (1983) 429.

[10] K. Benakli, Quelques aspects de la brisure de la supersymétrie en théorie des cordes, Ph.D Thesis, 1994; M. Dine and A. E. Nelson, Phys. Rev. D48 (1993) 1277.

[11] L.E. Ibáñez and C. López, Phys. Lett. 126B (1983) 54; Nucl. Phys. B233 (1984) 511.

[12] I. Antoniadis, J. Ellis, S. Kelley and D.V. Nanopoulos, Phys. Lett. 272B (1991) 31.

[13] J. Ellis, S. Kelley and D.V. Nanopoulos, Phys. Lett. 249B (1990) 441 and 260B (1991) 131; U. Amaldi, W.de Boer and H. Fürstenau, Phys. Lett. 260B (1991) 447; P. Langacker and M. Luo, Phys. Rev. D44 (1991) 817.

[14] V.S. Kaplunovsky, Nucl. Phys. B307 (1988) 145 and Errata STANFORD- 
ITP-838 preprint (1992).

[15] S. Bethke, XXVI International Conference on High Energy Physics, Dallas, August 1992.

[16] D. Bailin and A. Love, Mod. Phys. Lett. A7 (1992) 1485. 